\title{
Endocrine System Findings Domain
}

National Cancer Institute

\section{Source}

National Cancer Institute. Endocrine System Findings Domain. NCI Thesaurus. Code C102630.

A subject domain utilized for the submission of information encompassing and representing data, vocabulary or records related to endocrine system findings. 